\title{
Estudo Exploratório sobre a Violência entre Irmãos em Portugal
}

\author{
An Exploratory Study of Sibling Violence in Portugal
}

\author{
Inês Carvalho Relva* ${ }^{* a, b}$, Otília Monteiro Fernandes ${ }^{b}$, Madalena Alarcão $^{a}$ \\ \& Amadeu Quelhas Martins ${ }^{c}$ \\ ${ }^{a}$ Universidade de Coimbra e Centro de Estudos Sociais,Coimbra, Distrito de Coimbra, Portugal, \\ ${ }^{b}$ Universidade de Trás-os-Montes e Alto Douro, Vila Real, Distrito de Vila Real, Portugal \\ $\&{ }^{c}$ University of Cambridge, Cambridge, $U K$
}

\begin{abstract}
Resumo
A violência entre irmãos é uma das formas mais comuns de violência familiar. Este estudo pretendeu caracterizar este problema em Portugal, numa amostra de 588 estudantes universitários. Os diferentes tipos de violência (física, psicológica e sexual) foram avaliados com as "Revised Conflict Tactics Scales - Sibling Version". Procedeu-se a análises descritivas exploratórias das distribuições das principais variáveis, recorreu-se ao teste do $\chi^{2}$ e a análises de covariância múltipla. Os resultados indicaram que a violência entre irmãos é muito frequente no início da adolescência; os rapazes perpetraram significativamente mais atos de violência física $(p<0,001)$ e sexual $(p<0,05)$ do que as raparigas, mas foram também, física $(p<0,001)$ e sexualmente $(p<0,05)$, mais vitimizados. Também as díades masculinas apresentaram níveis de violência física e sexual significativamente superiores aos de todas as outras díades, indicando claramente a reciprocidade do fenómeno. Os resultados foram discutidos segundo perspetivas de 'normalização de agressões' (dissonância cognitiva; Hardy, Beers, Burguess, \& Taylor, 2010) e do 'ciclo de violência', as quais explicam a manutenção de comportamentos abusivos entre irmãos e a sua possível transferência para outras relações.

Palavras-chave: Violência, irmãos, díades fraternas, diferenças de género.
\end{abstract}

\begin{abstract}
Sibling violence is one of the most common forms of family violence. This study aimed to characterize this issue in Portugal, among a sample of 588 undergraduates. The different types of victimization (physical, psychological and sexual) were assessed through the "Revised Conflict Tactics Scale - Sibling Version". Descriptive exploratory analyses of the main variables, $\chi 2$ test, and multiple analyses of covariance were conducted. The results indicated that sibling violence is more frequent during early adolescence; boys committed significantly more physical $(p<.001)$ and sexual $(p<.05)$ violent acts than girls, but they were also significantly more victimized than girls, both physically $(p<.001)$ and sexually $(p<.05)$. Also, the male dyads showed significantly higher levels of physical and sexual violence than all other dyads, indicating clearly the reciprocal phenomenon. The results are discussed according to 'normalization of aggression' and 'cycle of violence' perspectives, which both help to understand the perpetration of abusive behaviors among siblings, as well as their occasional transfer to other relationships.

Keywords: Violence, siblings, fraternal dyads, gender differences.
\end{abstract}

A violência na família é um tema de destaque nos mídia e uma realidade preocupante na sociedade em geral. Formas de violência como a conjugal ou o abuso infantil atraem simultaneamente visibilidade académica (ex., Kiselica \& Morrill-Richards, 2007; Straus, Gelles, $\&$ Steinmetz, 1980) e mediatismo. Contudo, é raramente referida a violência exercida entre irmãos, embora surpreendentemente, uma vez que se acumulam evidências

\footnotetext{
* Endereço para correspondência: Departamento de Educação e Psicologia, Universidade de Trás-os-Montes e Alto Douro, Quinta de Prados, 5001-801, Vila Real, Portugal.E-mail: irelva@utad.pt, tila@utad.pt,madalena. alarcao@uc.pte aq219@cam.ac.uk
}

de que é a forma mais comum de violência na família (ex., Eriksen \& Jensen, 2009; Goodwin \& Roscoe, 1990; Wiehe, 1997) e, simultaneamente, a que origina maiores taxas de vitimização durante a infância e adolescência (ver Finkelhor, Turner, \& Ormond, 2006).

A maioria dos estudos sobre a fratria, desde os clássicos e pioneiros de Adler (1930/1993) e Toman (1959, 1993) até aos mais recentes de Bank e Kahn (1997) e Dunn (1983), tem-se debruçado sobretudo sobre as variáveis da constelação fraternal e o seu papel positivo (ex., protetor, orientador, facilitador de competências psicossociais e cognitivas) no desenvolvimento de cada um dos irmãos. É inquestionável a influência dos irmãos enquanto agentes socializadores (Fernandes, Alarcão, \& Raposo, 2007; Fer- 
Relva, I. C., Fernandes, O. M., Alarcão, M. \& Martins, A. Q. (2014). Estudo Exploratório sobre a Violência entre Irmãos em Portugal.

reira \& Mettel, 1999), sendo a fratria considerada mesmo como o primeiro laboratório social (Minuchin, 1974/1982). No entanto, esta complexa e duradoura relação pode ser também muito perturbadora, se pautada pela violência.

Como em qualquer relação interpessoal, a violência entre irmãos pode ter expressão física, psicológica, e/ou sexual. A violência física ocorre quando um irmão causa deliberadamente ferimentos ou a morte de um outro. São comportamentos que a ilustram: empurrar, puxar cabelos, arranhar, beliscar, pontapear e agredir com objetos (Wiehe, 1997) ou manusear armas (Caffaro \& Conn-Caffaro, 1998). A violência psicológica inclui comportamentos de negligência, atitudes e comentários depreciativos para ridicularizar, ameaçar ou aterrorizar o irmão, ou mesmo explorar a sua propriedade pessoal (Caffaro \& Conn-Caffaro, 1998). A violência ou abuso sexual entre irmãos não tem ainda definição consensual. Contudo, Caffaro e Conn-Caffaro (1998) consideram-na como um comportamento sexual para o qual a vítima não está preparada ao nível desenvolvimental, e, não sendo transitório nem motivado pela curiosidade própria da idade do abusador, pode envolver contacto físico, coerção ou força.

\section{Prevalência e Incidência de Violência entre Irmãos}

\section{Violência Física e Psicológica}

As experiências de vitimização física e psicológica entre irmãos foram exploradas por alguns autores, sobretudo nos EUA. Steinmetz (1977) encontrou taxas de prevalência de violência física superiores a $70 \%$. Estudos subsequentes confirmaram índices elevados de comportamentos abusivos entre irmãos, particularmente durante a infância (Goodwin \& Roscoe, 1990; Roscoe, Goodwin, \& Kennedy, 1987). Mais recentemente, Hardy, Beers, Burgess e Taylor (2010) verificaram, numa amostra de estudantes universitários, que mais de $75 \%$ dos participantes, independentemente do género, tinham perpetrado e sofrido atos de violência física e emocional entre irmãos, durante a infância. De um modo geral, a frequência dos episódios de violência parece decrescer com a entrada na adolescência, quer por resultado de maturação de competências relacionais, quer pelo menor tempo despendido no contacto entre irmãos (Noland, Liller, McDermott, Coutler, $\&$ Seraphine, 2004).

\section{Violência ou Abuso Sexual}

As interações sexuais entre irmãos podem decorrer de uma relação desigual de poder. Esses episódios de abuso têm, frequentemente, uma manutenção duradoura envolta em secretismo e manipulação (Canavan, Meyer, \& Higgs, 1992). Quase invariavelmente, os pais desvalorizam o comportamento sexual que ocorre entre irmãos, atribuindo-lhe um rótulo de "inocência" (Smith \& Israel, 1987), mas, quando sucede, o abuso sexual entre irmãos não difere das experiências de abuso perpetradas por pais ou padrastos (Cyr, Wright, McDuff, \& Perron, 2002). Na realidade, alguns autores acreditam que o abuso sexual entre irmãos pode ser mais prevalente que o abuso sexual parental (Kiselica \& Morrill-Richards, 2007; Welfare, 2008) e estar na origem de quase $50 \%$ dos casos de incesto (Laurance, 2000).

\section{Consequências da Violência entre Irmãos}

\section{Violência Física e Psicológica}

A violência entre irmãos produz consequências adversas em diversas áreas da vida das vítimas. A curto prazo, os irmãos vitimizados apresentam dificuldades académicas e problemas de comportamento com maior frequência que os irmãos não-vitimizados (Caffaro \& Conn-Caffaro, 1998; Duncan, 1999; Snyder, Bank, \& Burraston, 2005). Múltiplos problemas emocionais, como autoestima fragilizada, depressão, ou perturbações alimentares, têm sido também assinalados (Canavan et al., 1992; Wiehe, 1997). Em adolescentes, a experiência de vitimização propicia o uso de violência dirigida aos pares e parceiros afetivos (Noland et al., 2004; Rothman, Johnson, Azrael, Hall, \& Weinberg, 2010; Simonelli, Mullis, Elliot, \& Pierce, 2002), o envolvimento em comportamentos pré-delinquentes, como o uso de drogas ou a posse de armas (Rothman et al., 2010), ou manifestamente delinquentes (Button \& Gealt, 2010; Garcia, Shaw, Winslow, \& Yaggi, 2000). Na idade adulta, as vítimas evidenciam mais problemas relacionais com os seus irmãos (Bank \& Khan, 1997), mas também com os companheiros afetivos (Noland et al., 2004; Simonelli et al., 2002).

\section{Violência ou Abuso Sexual}

$\mathrm{O}$ abuso sexual entre irmãos pode perturbar o relacionamento intrafamiliar e afetar a vítima, na sua identidade e desenvolvimento sexual, e no seu funcionamento intrapsíquico (Adler \& Schutz, 1995). O abuso sexual na fratria irmão-irmã possui características semelhantes às do abuso sexual pai-filha quanto à ameaça ou uso da força, ou às sequelas físicas provocadas (Rudd \& Herzeberger, 1999). Na vida adulta das vítimas, múltiplos problemas de ajustamento (depressão, abuso de drogas ou álcool, perturbação de stresse pós-traumático e promiscuidade sexual) têm sido identificados (Canavan et al., 1992; Cyr et al., 2002; Rudd \& Herzberger, 1999). No geral, as consequências a longo prazo da vitimização sexual por irmãos serão tão mais devastadoras quanto maior a diferença de idades entre eles, a cronicidade e extensão do abuso, o uso de agressão e violência, e a ausência de comunicação intra e extrafamiliar (Finkelhor, 1980).

\section{Perspetivas Explicativas da Violência entre Irmãos}

\section{Violência Física e Psicológica}

Hoffman e Edwards (2004) evocam as teorias feminista, do conflito e da aprendizagem social para explicar estes tipos de violência. A primeira assenta na organização patriarcal da sociedade, em que os homens têm acesso privilegiado aos recursos (Bograd, 1990). Nesta visão, 
os irmãos (rapazes) tornam-se perpetradores de violência à semelhança dos pais (homens) (Finkelhor, 1980). Frequentemente, os irmãos utilizam a vantagem física sobre as irmãs para controlar o seu comportamento e demonstrar a sua masculinidade (Wiehe, 1997), o mesmo ocorrendo em relação aos irmãos mais novos e fisicamente menos resistentes. Já a teoria do conflito assume a agressão e a violência como meios de resolução de conflitos em contexto familiar (Felson \& Tedeschi, 1993). A violência na díade fraterna funcionaria como veículo de expressão de raiva devido a favoritismo parental, ou a conflitos resultantes de obrigações domésticas (Hoffman \& Edwards, 2004). Por seu lado, a teoria da aprendizagem social postula que a violência física e psicológica entre irmãos é aprendida por imitação e reforço, a partir de modelos que as interações negativas entre pais e entre pais e filhos fornecem às crianças (Caffaro \& Conn-Caffaro, 1998; Noller, 2005). Esses comportamentos seriam posteriormente replicados pelos filhos em situações similares, favorecendo a transmissão intergeracional da violência na família (Wiehe, 1997).

Globalmente, estas teorias avançam explicações etiológicas para o fenómeno, mas não para a sua severidade/ frequência. Para tal compreensão devem ser considerados fatores inerentes ao contexto familiar e às características particulares de vítimas e perpetradores. A indisponibilidade parental ou a falta de competências na supervisão das crianças, os desequilíbrios de poder entre os irmãos, a indefinição de fronteiras dos seus espaços físicos e psicológicos, a par de tratamento diferencial por parte dos pais, têm sido associados a episódios mais frequentes ou mais graves de violência entre irmãos (Caffaro \& Conn-Caffaro, 1998; Wiehe, 1997). Outras variáveis sistémicas implicadas são as situações de stresse familiar, como sejam as dificuldades financeiras ou a doença (Hardy, 2001).

\section{Violência ou Abuso Sexual}

As principais teorias explicativas do abuso sexual entre irmãos assentam sobretudo em particularidades dos perpetradores. A "teoria do bloqueio" postula que os abusadores têm um marcado défice de competências sociais e interpessoais que os impede de cultivar relações significativas com os pares e os impele a satisfazer as suas necessidades com irmãos (irmãs) mais jovens, naturalmente vulneráveis (O’Brien, 1991). Uma outra perspetiva é conhecida como "síndrome do vampiro", por remeter a explicação do abuso sexual entre irmãos para um padrão intergeracional de abuso. Esta visão encontra suporte empírico no número de abusadores que foram eles próprios vítimas de abuso sexual pelos familiares diretos (Smith \& Israel, 1987). Inquestionavelmente, certas características intrafamiliares, sugestivas de algum grau de disfunção, propiciam a ocorrência de abuso sexual entre irmãos. Frequentemente, este surge no contexto de relações de poder ou de enorme dependência, sendo o perpetrador encoberto por um certo favoritismo parental (Caffaro \& Conn-Caffaro, 1998; Russell, 1986). Tais aspetos relacionais, associados à vergonha, culpa, repressão, ou mesmo dissociação, contribuem para o silêncio das vítimas (Wiehe, 1997). Também a exposição à atividade sexual de familiares (O'Brien, 1991), ou o acesso a materiais pornográficos (Seto \& Lalumière, 2010), parece desempenhar uma função etiológica no fenómeno.

Em suma, a violência entre irmãos é uma realidade muito comum, que pode originar sequelas emocionais e psicossociais duradouras, mas paradoxalmente tem sido vista como trivial e está mal quantificada. Em Portugal, são ainda desconhecidos dados sistemáticos que a caracterizem, pelo que o presente estudo é pioneiro em pretender: (a) Estimar a ocorrência de vários tipos de violência (i.e., emocional, física, e sexual) entre irmãos numa amostra de estudantes universitários; (b) Identificar os comportamentos abusivos/tipos de vitimização mais frequentes por género; e (c) Avaliar a influência das características das fratrias na severidade desses comportamentos.

\section{Método}

\section{Participantes}

Quinhentos e oitenta e oito estudantes universitários (219 do sexo masculino), com idade média de 20 anos ( \pm 4), todos com irmãos, consentiram em participar no estudo. Cerca de $65 \%$ da amostra possuía apenas um irmão, 24\% tinha dois, $7 \%$ três, e os restantes $4 \%$ tinham quatro ou mais. Foi solicitado aos participantes que indicassem o sexo do irmão com quem experimentaram mais conflitos durante o período de referência etária dos 13 anos de idade. De igual modo, solicitou-se que indicassem a idade que o irmão tinha nesse período. Com base nestas informações, determinou-se a composição das díades fraternas e a posição do participante na fratria.

\section{Instrumentos}

Questionário Sociobiográfico (QSB). Questionário de informação sociodemográfica adaptado do Social Environment Questionnaire (Toman, 1993), que incide sobre características do sujeito, da sua família e do subsistema fraternal.

Revised Conflict Tactics Scales - Sibling Version (CTS2-SP; Straus, Hamby, Boney-McCoy, \& Sugarman, 1996). Questionário de 39 itens já aferido para a população portuguesa (Relva, Fernandes, \& Costa, 2013) que permite avaliar as táticas de conflito preferencialmente utilizadas no relacionamento entre irmãos. Como as CTS2-SP se cingem à conflitualidade na díade fraterna, quando o sujeito tem mais do que um irmão é convidado a responder em relação ao que lhe é mais próximo em idade. Este instrumento é composto por cinco escalas: negociação (6 itens), agressão psicológica ( 8 itens; nesta escala decidiu excluir-se um dos itens porque na validação portuguesa das CTS2-SP (Relva et al., 2013) todos os itens apresentavam uma correlação item-total acima de 0,30 , exceto um, pelo que todas as análises foram feitas considerando apenas 7, e não 8 itens), agressão física sem sequelas (12 itens), coerção sexual (7 itens) e agressão 
Relva, I. C., Fernandes, O. M., Alarcão, M. \& Martins, A. Q. (2014). Estudo Exploratório sobre a Violência entre Irmãos em Portugal.

física com sequelas (6 itens). Cada item é representado por duas questões, a primeira referindo um determinado comportamento emitido pelo sujeito em relação ao irmão/ irmã, e a segunda referindo o mesmo comportamento em sentido inverso (do irmão/irmã em relação ao sujeito). O questionário começa por avaliar táticas de conflito convencionais (ex., negociação), passando gradualmente para comportamentos mais coercivos e desaprovados socialmente. A escala de resposta traduz a frequência de cada comportamento num determinado período de tempo: de 0 ("nunca aconteceu") a 7 ("não naquele ano, mas já aconteceu"). A escala de negociação não foi utilizada na presente investigação, uma vez que o objeto de estudo era a violência entre irmãos, isto é, o uso de táticas de conflito coercivas nas díades fraternas.

\section{Procedimentos}

Após obtenção de autorizações institucionais, os questionários, anónimos, foram autoadministrados em turmas de diferentes universidades portuguesas. $\mathrm{O}$ seu preenchimento foi voluntário e sem incentivos. O horário de administração foi previamente acordado com os responsáveis das turmas, normalmente antes ou depois das aulas. Foram explicitados os objetivos do estudo em cada turma e realizou-se o briefing dos participantes após a recolha dos instrumentos. Para evitar homogeneidade amostral, os participantes foram recrutados em cursos de diferentes áreas de conhecimento.

Procedimentos de Análise e de Redução de Dados. Conduziram-se análises descritivas exploratórias (coeficientes de assimetria, curtose e inspeção de "boxplots") das distribuições das principais variáveis. A determinação das prevalências dos vários tipos de violência perpetrada/ sofrida foi acompanhada da estimativa de intervalos de confiança a 95\% para proporções segundo o método de aproximação da distribuição binomial à normal. A análise das frequências dos itens das CTS2-SP recorreu ao teste do $\chi^{2}$ com a correção de Yates. Os somatórios das pontuações nas escalas das CTS2-SP constituíram três pontuações totais de severidade de violência, a saber, psicológica (escala de "agressão psicológica"), física (escalas de "agressão física sem sequelas" e "agressão física com sequelas"), e sexual (escala de "coerção sexual"), respetivamente, para perpetradores e para vítimas. Os vários tipos de violência perpetrada e/ou sofrida foram comparados por género através do teste $t$ de Student para amostras independentes. O $d$ de Cohen, medida de magnitude do efeito (Cohen, 1992), é reportado em cada comparação efetuada. Cada díade fraterna foi classificada a partir da combinação "sexo do participante/sexo do irmão", constituindo a variável "composição da díade". O estudo da severidade de violência perpetrada/sofrida nas díades efetuou-se por análises de covariância múltipla (MANCOVAs) das pontuações totais de violência para cada composição de díade, controlando a diferença absoluta de idades entre os irmãos (covariável). Seguindo as recomendações de Field (2009) e de Peat e Barton (2005), os pressupostos da MANCOVA foram testados a partir da distância de Mahalanobis, de coeficientes de correlação de Pearson, do teste $\mathrm{M}$ de Box, e da análise da interação entre a covariável e cada variável dependente (homogeneidade do declive da reta de regressão). A violação do pressuposto da homogeneidade da matriz de covariâncias (testes $\mathrm{M}$ de Box com $p<0,05$ ) implicou a ponderação da estandardização das pontuações totais de violência (escores $Z$ ), dada a marcada assimetria das distribuições das pontuações totais de violência (curva "L-shaped"), pois com a sua transformação obter-se-ia a normalização mais eficaz através do uso da recíproca de cada variável seguindo a fórmula "1 / (variável +1)"; no entanto, optou-se por não seguir esta transformação pois comprometeria a compreensão dos resultados em testes post-hoc; por outro lado, utilizámos o uso do traço de Pillai (em vez do $\Lambda$ de Wilks) para avaliação da significância multivariada dos efeitos (Tabachnick \& Fidell, 2007). O eta-quadrado $\left(\eta_{p}^{2}\right)$ parcial multivariado $\left(\eta_{p}^{2}=1-V\right.$ Pillai ${ }^{1 / s}$ ) foi considerado como medida de magnitude dos efeitos. O seguimento de efeitos significativos realizou-se através de testes post-hoc com ajustamento de Bonferroni para comparações múltiplas. O tratamento estatístico decorreu com o Statistical Package for the Social Sciences (versão 18).

\section{Resultados}

\section{Violência Psicológica, Física e Sexual}

Cerca de $88 \%$ (intervalo de confiança a $95 \%=[85,8 \%$ $-91,0 \%]$ ), $69 \%$ (intervalo de confiança a $95 \%=[65,5 \%$ $-72,9 \%]$ ), e $6 \%$ (intervalo de confiança a $95 \%=[4,3 \%$ - 8,3\%]) dos participantes admitiram ter perpetrado, respetivamente, atos de violência psicológica, física e sexual aos seus irmãos durante o período de referência dos 13 anos de idade. Comparativamente, as frequências de vitimização psicológica, física e sexual de irmãos para o mesmo período foram, respetivamente, $87 \%$ (intervalo de confiança a $95 \%=[84,5 \%-89,9 \%]), 69 \%$ (intervalo de confiança a $95 \%=[65,5 \%-72,9 \%]$ ), e $8 \%$ (intervalo de confiança a $95 \%=[5,4 \%-9,6 \%])$. As taxas de perpetração e de vitimização dos participantes foram muito coincidentes, pelo que se estimou a taxa de coocorrência de perpetração e vitimização para cada tipo de abuso: $87 \%$ (intervalo de confiança a $95 \%=[83,9 \%-89,4 \%]$ ), $66 \%$ (intervalo de confiança a $95 \%=[61,7 \%-69,3 \%]$ ) e $4 \%$ (intervalo de confiança a $95 \%=[2,1 \%-5,1 \%]$ ) dos participantes admitiram ter simultaneamente perpetrado e sofrido, respetivamente, atos de violência psicológica, física e sexual na relação fraterna durante esse período.

\section{Comparação, por Género, da Violência Perpetrada e Sofrida}

A Tabela 1 apresenta as pontuações totais das CTS2-SP para cada forma de violência perpetrada ou sofrida entre irmãos, separadamente, por género. 
Psicologia: Reflexão e Crítica, 27(2), 398-408.

Tabela 1

Comparação, por Género, das Pontuações Totais das CTS2-SP Reportadas pelos Participantes

\begin{tabular}{lcccccc}
\hline Pontuações totais CTS2-SP & $\begin{array}{c}\text { Género Masculino } \\
n=219 \\
(\text { Média } \pm D P)\end{array}$ & $\begin{array}{c}\text { Género Feminino } \\
n=369 \\
\text { Média } \pm D P)\end{array}$ & $t$ & $g l$ & $p$ & $d$ \\
\hline Tipos de violência perpetrada & & & & & & \\
Psicológica & $11,7(9,7)$ & $10,3(8,2)$ & 1,87 & 399,8 & 0,06 & 0,19 \\
Física & $10,9(14,9)$ & $6,1(8,7)$ & 4,35 & 306,9 & $<0,001$ & 0,50 \\
Sexual & $1,1(4,1)$ & $0,2(1,0)$ & 3,21 & 233,5 & 0,002 & 0,42 \\
Tipos de violência sofrida & & & & & & \\
Psicológica & $11,3(9,6)$ & $10,1(8,3)$ & 1,60 & 406,8 & 0,11 & 0,16 \\
Física & $10,5(14,8)$ & $6,4(8,4)$ & 3,76 & 301,9 & $<0,001$ & 0,43 \\
Sexual & $1,1(4,3)$ & $0,2(1,0)$ & 3,04 & 232,5 & 0,003 & 0,40 \\
\hline
\end{tabular}

Nota. CTS2-SP = Revised Conflict Tactics Scales - Sibling Version (Portuguese); DP = Desvio-padrão; $g l=$ Graus de liberdade; $d$ $=d$ de Cohen, estimado a partir dos valores de $t$ e dos respectivos graus de liberdade. $N=588$.

Perpetração de Violência. O recurso à violência psicológica foi ligeiramente mais reportado pelos rapazes; no entanto, esta diferença não atingiu significância estatística $(p=0,06)$. Já o recurso à violência "física" $(p<0,001) \mathrm{e}$ "sexual" $(p<0,05)$ foi significativamente mais frequente entre os rapazes.

Vitimização de Violência. Não ocorreram diferenças de género quanto à experiência de violência psicológica sofrida pelos irmãos $(p=0,11)$. No entanto, os participantes masculinos referiram níveis de vitimização "física" $(p$ $<0,001)$ e "sexual" $(p<0,05)$ significativamente superiores aos dos participantes femininos.

\section{Comportamentos de Vitimização mais Frequentes por Género}

A Tabela 2 descreve os comportamentos abusivos sofridos pelos participantes e reportados nas CTS2-SP para cada forma de violência entre irmãos. As respetivas frequências foram comparadas por género, separadamente, para respostas de "vitimização".

Perpetração de Violência. Relativamente à forma psicológica de violência, os atos mais comuns perpetrados pelos participantes contra os seus irmãos foram, os insultos, os gritos e as provocações. À exceção da maior tendência $(p<0,10)$ dos rapazes em usar comentários depreciativos, nenhuma diferença de género foi detetada. Quanto aos comportamentos de violência física, os rapazes revelaram frequências significativamente aumentadas de perpetração de vários atos de violência com sequelas físicas (provocar fraturas, desmaios ou atendimento hospitalar) e sem sequelas físicas (atirar objetos, torcer o braço/puxar o cabelo, agarrar à força, usar uma faca ou arma, esmurrar ou bater, tentar sufocar o irmão, entre outros). Apesar destas diferenças, comportamentos como atirar objetos, torcer o braço ou puxar o cabelo, empurrar ou apertar, e dar uma bofetada parecem ser comuns entre irmãos. Finalmente, diversos comportamentos sexualmente abusivos (forçar relações sexuais sem preservativo, insistir em ter relações sexuais ou sexo oral/anal, usar a força para ter relações sexuais, recorrer a ameaças para ter relações sexuais ou sexo oral/anal) foram também significativamente mais frequentes entre os rapazes.

Vitimização de Violência. À semelhança dos resultados descritos para a violência psicológica perpetrada, os insultos, os gritos e as provocações foram os atos abusivos mais comuns que os participantes, independentemente do género, sofreram dos seus irmãos. Os rapazes foram, no entanto, alvos significativamente mais frequentes $(p<0,05)$ de ameaças. Considerando os atos de violência física, os rapazes foram significativamente mais vitimizados por alguns comportamentos com sequelas físicas (provocar desmaios ou atendimento hospitalar) e sem sequelas físicas (atirar objetos, torcer o braço/puxar o cabelo, agarrar à força, usar uma faca ou arma, esmurrar ou bater, tentar sufocar o irmão, entre outros). Uma vez mais, atirar objetos, torcer o braço ou puxar o cabelo, e empurrar ou apertar revelaram-se comuns entre as vítimas, apesar da diferença de género assinalada para os dois primeiros comportamentos. Por fim, e algo surpreendentemente, também os rapazes foram as vítimas significativamente mais frequentes para a maioria dos comportamentos sexualmente abusivos.

\section{Influência das Características das Díades Fraternas na Severidade dos Vários Tipos de Violência}

Violência Perpetrada. O cálculo exploratório de correlações bivariadas indicou que a diferença absoluta de idade entre os irmãos era a única variável sociodemográfica significativamente associada às pontuações totais de violência psicológica $(r=-0,26 ; p<0,001)$ e física $(r=-0,20$; $p<0,001$ ), pelo que esta foi controlada na análise. Uma MANCOVA foi conduzida nas notas estandardizadas de violência perpetrada (psicológica, física e sexual) para a 
Relva, I. C., Fernandes, O. M., Alarcão, M. \& Martins, A. Q. (2014). Estudo Exploratório sobre a Violência entre Irmãos em Portugal.

Tabela 2

Comparação, por Género, das Frequências de Comportamentos Violentos Sofridos Pelos Participantes

\begin{tabular}{|c|c|c|c|c|c|}
\hline Itens CTS2-SP & $\begin{array}{c}\text { Género } \\
\text { Masculino } \\
n(\%)\end{array}$ & $\begin{array}{c}\text { Género } \\
\text { Feminino } \\
n(\%)\end{array}$ & $\chi^{2}$ & $R$ & $\mathrm{IC}_{95 \%}$ \\
\hline \multicolumn{6}{|l|}{ Violência Psicológica } \\
\hline Insultar ou dizer palavrões & $161(73,5)$ & $267(72,4)$ & 0,04 & 1,06 & $0,73-1,55$ \\
\hline Gritar ou berrar & $162(74,0)$ & $275(74,5)$ & 0,03 & 0,97 & $0,66-1,42$ \\
\hline Sair abruptamente da sala & $95(43,4)$ & $173(46,9)$ & 0,55 & 0,87 & $0,62-1,22$ \\
\hline Fazer algo para irritar & $124(56,6)$ & $229(62,1)$ & 1,48 & 0,80 & $0,57-1,12$ \\
\hline Chamar de gordo/a ou feio/ a & $79(36,1)$ & $125(33,9)$ & 0,20 & 1,10 & $0,78-1,56$ \\
\hline Destruir algo & $91(41,6)$ & $142(38,5)$ & 0,42 & 1,14 & $0,81-1,60$ \\
\hline Ameaçar ferir ou atirar algo & $61(27,9)$ & $73(19,8)$ & $4,64^{*}$ & 1,57 & $1,06-2,31$ \\
\hline \multicolumn{6}{|l|}{ Violência Física } \\
\hline Atirar alguma coisa & $105(47,9)$ & $142(38,5)$ & $4,67^{*}$ & 1,47 & $1,05-2,07$ \\
\hline Torcer o braço ou puxar o cabelo & $104(47,5)$ & $141(38,2)$ & $4,49^{*}$ & 1,46 & $1,04-2,05$ \\
\hline Empurrar ou apertar & $100(45,7)$ & $170(46,1)$ & 0,01 & 0,98 & $0,70-1,38$ \\
\hline Agarrar à força & $55(25,1)$ & $52(14,1)$ & $10,49^{* *}$ & 2,04 & $1,34-3,12$ \\
\hline Dar uma bofetada & $84(38,4)$ & $139(37,7)$ & 0,01 & 1,03 & $0,73-1,45$ \\
\hline Usar uma faca ou uma arma & $14(6,4)$ & $5(1,4)$ & $9,60 * *$ & 4,97 & $1,77-14,00$ \\
\hline Esmurrar ou bater & $49(22,4)$ & $48(13,0)$ & $8,09 * *$ & 1,93 & $1,24-2,99$ \\
\hline Tentar sufocar & $18(8,2)$ & $13(3,5)$ & $5,17 *$ & 2,45 & $1,18-5,11$ \\
\hline Atirar contra a parede & $38(17,4)$ & $38(10,3)$ & $5,47 *$ & 1,83 & $1,13-2,97$ \\
\hline Dar uma tareia & $36(16,4)$ & $38(10,3)$ & $4,17^{*}$ & 1,71 & $1,05-2,80$ \\
\hline Queimar ou escaldar & $11(5,0)$ & $1(0,3)$ & $13,24 * *$ & 19,46 & $2,50-151,81$ \\
\hline Dar pontapés & $76(34,7)$ & $101(27,4)$ & $3,17^{\dagger}$ & 1,41 & $0,98-2,02$ \\
\hline Provocar entorse, ferida ou pequeno corte & $78(35,6)$ & $111(30,1)$ & 1,69 & 1,29 & $0,90-1,83$ \\
\hline Provocar dor física, que se manteve no dia seguinte & $32(14,6)$ & $39(10,6)$ & 1,75 & 1,45 & $0,88-2,39$ \\
\hline Provocar desmaio & $11(5,0)$ & $3(0,8)$ & $8,75^{* *}$ & 6,45 & $1,78-23,39$ \\
\hline Provocar ida ao médico & $18(8,2)$ & $8(2,2)$ & $10,52 * *$ & 4,04 & $1,73-9,46$ \\
\hline Provocar ida ao médico, mas não ter ido & $17(7,8)$ & $12(3,3)$ & $5,04 *$ & 2,50 & $1,17-5,35$ \\
\hline Provocar fratura & $13(5,9)$ & $10(2,7)$ & $3,00^{\dagger}$ & 2,27 & $0,98-5,26$ \\
\hline \multicolumn{6}{|l|}{ Violência Sexual } \\
\hline Forçar a relações sexuais sem preservativo & $16(7,3)$ & $11(3,0)$ & $4,92 *$ & 2,57 & $1,17-5,63$ \\
\hline Insistir em ter relações sexuais & $7(3,2)$ & $1(0,3)$ & $6,72 * *$ & 12,15 & $1,49-99,44$ \\
\hline Insistir em ter sexo oral/ anal & $8(3,7)$ & $1(0,3)$ & $8,31 * *$ & 13,95 & $1,73-112,33$ \\
\hline Usar a força para ter relações sexuais & $12(5,5)$ & $3(0,8)$ & $10,24 * *$ & 7,07 & $1,97-25,35$ \\
\hline Usar a força para ter sexo oral/ anal & $10(4,6)$ & $1(0,3)$ & $11,57 * *$ & 17,61 & $2,24-138,51$ \\
\hline Recorrer a ameaças para ter sexo oral/ anal & $10(4,6)$ & $5(1,4)$ & $4,48^{*}$ & 3,48 & $1,18-10,33$ \\
\hline Recorrer a ameaças para ter relações sexuais & $9(4,1)$ & $6(1,6)$ & 2,48 & 2,59 & $0,91-7,39$ \\
\hline
\end{tabular}

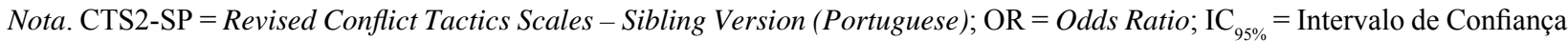
a $95 \% . N=588$.

${ }^{\dagger} p<0,10 ; * p<0,05 ; * * p<0,01$.

composição das díades, controlando a diferença absoluta de idade entre os irmãos, e indicou um efeito multivariado da composição das díades na combinação linear das pontuações de violência, $\mathrm{V}^{\text {Pillai }}=0,08, F(9,1719)=4,88$, $p<0,001, \eta^{2}=0,03$. Testes univariados confirmaram um efeito da composição das díades para as pontuações de violência física, $F(3,573)=10,78, p<0,001, \eta^{2}=0,05$, e sexual, $F(3,573)=8,23, p<0,001, \eta^{2}=0,04$, mas não 
Psicologia: Reflexão e Crítica, 27(2), 398-408.

para as pontuações de violência psicológica, $F(3,573)=$ $1,08, p=0,356, \eta^{2}<0,01$. A Tabela 3 apresenta as médias marginais estimadas para as pontuações de violência perpetrada em cada tipo de díade, quando controlada a diferença absoluta de idade entre os irmãos.

Tabela 3

Pontuações Estandardizadas de Violência Perpetrada pelos Participantes (CTS2-SP) em Cada Tipo de Díade, quando Controlada a Diferença Absoluta de Idade entre os Irmãos

\begin{tabular}{|c|c|c|c|c|}
\hline Pontuações totais de violência (notas z) & $\begin{array}{c}\text { Grupo 1 } \\
\text { Masculino/ } \\
\text { Masculino } \\
n=103\end{array}$ & $\begin{array}{c}\text { Grupo } 2 \\
\text { Masculino/ } \\
\text { Feminino } \\
n=112\end{array}$ & $\begin{array}{c}\text { Grupo } 3 \\
\text { Feminino/ } \\
\text { Masculino } \\
n=180\end{array}$ & $\begin{array}{c}\text { Grupo } 4 \\
\text { Feminino/ } \\
\text { Feminino } \\
n=183\end{array}$ \\
\hline \multicolumn{5}{|l|}{ Violência Psicológica } \\
\hline Média (EP) & $0,15(0,10)$ & $-0,02(0,09)$ & $-0,06(0,07)$ & $-0,02(0,07)$ \\
\hline IC 95\% & {$[-0,04 ; 0,34]$} & {$[-0,20 ; 0,16]$} & {$[-0,20 ; 0,09]$} & {$[-0,16 ; 0,12]$} \\
\hline \multicolumn{5}{|l|}{ Violência Física } \\
\hline Média (EP) & $0,47(0,09)$ & $-0,01(0,09)$ & $-0,10(0,07)$ & $-0,17(0,07)$ \\
\hline IC $95 \%$ & {$[0,29 ; 0,66]$} & {$[-0,18 ; 0,17]$} & {$[-0,24 ; 0,04]$} & {$[-0,31 ;-0,03]$} \\
\hline \multicolumn{5}{|l|}{ Violência Sexual } \\
\hline Média (EP) & $0,42(0,10)$ & $0,00(0,09)$ & $-0,10(0,07)$ & $-0,14(0,07)$ \\
\hline IC 95\% & {$[0,23 ; 0,61]$} & {$[-0,18 ; 0,18]$} & {$[-0,24 ; 0,05]$} & {$[-0,29 ; 0]$} \\
\hline
\end{tabular}

Nota. $\mathrm{EP}=$ Erro-padrão da média. $\mathrm{IC}_{95 \%}=$ Intervalo de Confiança a 95\%. $N=578$.

Testes post-hoc com ajustamento de Bonferroni indicaram pontuações de violência física significativamente superiores para as díades $\mathrm{M}-\mathrm{M}$, quando comparadas com díades M-F $(p<0,01)$, F-M $(p<0,001)$ e F-F $(p<0,001)$ [M de masculino e $\mathrm{F}$ de feminino]. De igual modo, as pontuações de violência sexual das díades $\mathrm{M}-\mathrm{M}$ foram substancialmente superiores às de díades M-F $(p=0,01)$, F-M $(p<0,001)$ e F-F $(p<0,001)$. Nenhuma outra diferença foi detetada.

Violência Sofrida. Como anteriormente, apenas a diferença absoluta de idade entre os irmãos se associou significativamente às pontuações totais de violência psicológica $(r=-0,28 ; p<0,001)$ e física $(r=-0,23 ; p$ $<0,001)$ sofridas. Através da MANCOVA, as pontuações estandardizadas de violência sofrida (psicológica, física e sexual) foram comparadas entre as diferentes díades, controlando novamente a diferença absoluta de idade entre os irmãos. Um efeito multivariado da composição das díades na combinação linear das pontuações de violência foi detetado, $\mathrm{V}^{\text {Pillai }}=0,08, F(9,1719)=5,28, p<0,001, \eta^{2}$ $=0,03$. Testes univariados indicaram que a composição das díades influenciou significativamente as pontuações de violência física, $F(3,573)=10,50, p<0,001, \eta^{2}=0,05$, e sexual, $F(3,573)=6,80, p<0,001, \eta^{2}=0,03$, mas não as de violência psicológica, $F(3,573)=0,97, p=0,406, \eta^{2}<0,01$.

Uma vez mais, as comparações post-hoc indicaram pontuações de vitimização física significativamente superiores entre as díades $\mathrm{M}-\mathrm{M}$, quando comparadas com as díades M-F $(p<0,01)$, F-M $(p<0,001)$ e F-F $(p<0,001)$. Relativamente à vitimização sexual, as pontuações das díades M-M foram também significativamente superiores às de díades M-F $(p<0,05), \mathrm{F}-\mathrm{M}(p<0,001)$ e F-F $(p$
$<0,001)$. Nenhuma outra diferença foi assinalada entre as diversas díades.

\section{Discussão}

A violência entre irmãos é provavelmente a forma de violência mais comum nas famílias (Goodwin \& Roscoe, 1990), mas, paradoxalmente, a mais ignorada. O presente estudo estimou, retrospetivamente, as frequências de violência psicológica, física e sexual entre irmãos, pela administração das escalas CTS2-SP a estudantes universitários, comparando-as, por género e em termos de violência sofrida e perpetrada. Adicionalmente, a violência entre irmãos foi analisada segundo a composição de género e diferenças de idade das díades fraternas. Os resultados obtidos indicam que a violência entre irmãos é altamente prevalente no início da adolescência, especificamente para a idade de referência dos 13 anos, sendo maioritariamente expressa por comportamentos de abusos psicológicos e físicos sem sequelas (cf. Hardy, 2001; Simonelli et al., 2002), comportamentos esses ora perpetrados ora sofridos particularmente pelos irmãos rapazes. Além disso, os resultados sugerem que a composição de género das fratrias e as idades aproximadas (cf. Hardy et al., 2010) parecem contribuir para a expressão do fenómeno da violência entre irmãos. No entanto, variáveis do contexto familiar como o tratamento diferencial por parte dos pais, a indisponibilidade parental, a par de outras variáveis sistémicas (ex., exposição à violência parental e conjugal, doença, problemas financeiros) não abordadas no presente estudo, deverão ser tidas em consideração na compreensão do fenómeno. 
As prevalências de violência psicológica e de violência física aqui reportadas aproximam-se dos valores indicados por diversos estudos internacionais (ex., Graham-Bermann, Cutler, Litzenberger, \& Schwartz, 1994; Roscoe et al., 1987). O presente estudo demonstra ainda que a coocorrência de violência perpetrada e de violência sofrida parece ser a "regra" na relação fraterna, indicando que a violência entre irmãos é um fenómeno claramente recíproco (cf. Krienert \& Walsh, 2011a). Paralelamente, as percentagens de violência sexual aqui reportadas (inferiores a 10\%) são também semelhantes às descritas pelos escassos estudos retrospetivos desta forma de abuso, nomeadamente $7 \%$ (Hardy, 2001), 13\% (Finkelhor, 1980) e 16\% (Russell, 1986). No entanto, é difícil o contraponto com a literatura existente; por um lado, nem sempre são recrutados participantes de ambos os sexos (Russell, 1986), por outro, as frequências variam substancialmente quando a amostragem assenta em casos de abuso sexual identificados em contextos clínicos ou judiciais (ex., Wiehe, 1997). De todo modo, o presente estudo testemunha que esta dimensão de abuso fez também parte da realidade intrafamiliar de jovens universitários portugueses, embora esta não seja uma amostra representativa da população portuguesa, mas talvez uma amostra de "elite" dado que apenas uma parte dos jovens frequentam o ensino superior em Portugal, o que pode levar a pressupor que estas frequências possam ser diferentes noutras franjas menos escolarizadas e de outro nível socioeconómico.

A comparação por género dos diferentes tipos de violência e perfis de comportamento abusivo merece diversas considerações. A violência psicológica revelou-se o tipo de abuso e de vitimização mais frequente entre irmãos independentemente do género, tal como o reportado noutros estudos (ex., Goodwin \& Roscoe, 1990; Noland et al., 2004). Adicionalmente, a diferença absoluta de idade entre os irmãos associou-se significativa e negativamente a este tipo de violência, sugerindo que as agressões verbais são uma "trivialidade" entre irmãos com idades aproximadas. Por seu lado, a perpetração de violência física foi significativamente mais comum entre os participantes masculinos, resultado compatível com evidências empíricas de que os rapazes recorrem mais frequentemente à violência física para resolver conflitos com os irmãos (Eriksen \& Jensen, 2006; Hoffman, Kiecolt, \& Edwards, 2005). Esta tendência pode refletir, por um lado, a necessidade dos rapazes serem mais agressivos e competitivos do que as raparigas, para que se adequem a expectativas de uma "sociedade de género" (Leder, 1993); por outro lado, se as práticas educativas parentais forem elas próprias diferenciadas, poderão também justificar esta diferença. Além disso, as punições físicas são mais frequentemente dirigidas aos rapazes, o que permite que a agressão física seja modelada entre irmãos (Lytton \& Romney, 1991). Tal visão é aqui parcialmente suportada pelo facto dos atos de violência física severa (ex., dar uma tareia, tentar sufocar o irmão, provocar fraturas, desmaios ou idas ao médico) terem sido sobretudo perpetrados por participantes masculinos. De igual modo, também os participantes do sexo masculino reportaram um número significativamente superior de vitimizações físicas, tratando-se de algo surpreendente e sobre o qual a literatura é pouco consistente. Há estudos que indicam que as raparigas são significativamente mais vitimizadas na relação fraterna (Goodwin \& Roscoe, 1990; Kettrey \& Emery, 2006; Krienert \& Walsh, 2011a), outros que não encontram diferenças de género nas vitimizações (Duncan, 1999; Hardy, 2001), e ainda outros que documentam os rapazes como vítimas mais frequentes de abusos físicos (Hardy et al., 2010; Noland et al., 2004). Estudos futuros deverão elucidar o motivo destas discrepâncias, mas influências metodológicas estarão certamente implicadas; como exemplo, não será por acaso que as raparigas apresentam significativamente mais vitimizações físicas quando a respetiva amostragem contabiliza queixas judiciais formalizadas contra irmãos (ex., Kettrey \& Emery, 2006; Krienert \& Walsh, 2011a). Esta aparente diferença de género poderá derivar mais da "dinâmica de género" no comportamento de denúncia (Krienert \& Walsh, 2011a) do que do fenómeno de violência per se, desconhecendo-se se está também implicada na forma sexual de violência entre irmãos.

No presente estudo, a perpetração de comportamentos sexualmente abusivos foi significativamente mais frequente entre os participantes masculinos, mas também o foram as experiências de vitimização sexual. De facto, parece consensual que os comportamentos de coerção sexual são mais frequentemente perpetrados por rapazes (cf. Caffaro \& Conn-Caffaro, 2005; Hardy, 2001), mas há um hiato na literatura quanto à distribuição das vitimizações, por género. Como a maior parte dos estudos que registam abusos sexuais por irmãos decorrem de amostras clínicas (Adler \& Schutz, 1995; Canavan et al., 1992), agrupam sobretudo casos de incesto irmão-irmã e raramente incluem vítimas (ou perpetradores) de ambos os sexos (Krienert \& Walsh, 2011b). Apesar disso, foram pontualmente encontradas prevalências superiores a $20 \%$ de vitimizações sexuais em participantes masculinos (Caffaro \& Conn-Caffaro, 2005; Krienert \& Walsh, 2011b), valor preocupante e eventualmente compatível com a "síndrome do vampiro", anteriormente referida, na explicação da perpetuação de abusos sexuais em díades masculinas.

A comparação entre díades, para os vários tipos de violência perpetrada/sofrida, permite várias considerações. Primeiro, a associação significativa da diferença absoluta de idade entre os irmãos com as formas psicológica e física de violência (quer perpetrada, quer sofrida) implicou o ajuste estatístico para este fator através da MANCOVA. Por um lado, alguns estudos sugerem que as agressões físicas são mais frequentes entre irmãos com diferença de idade inferior a três anos (ex., Felson \& Russo, 1988; Straus et al., 1980). Por outro, a idade é um confounder muito recorrente no estudo de díades (Krienert \& Walsh, 2011a), particularmente porque as díades são o produto do desenvolvimento de cada um dos irmãos e estão sujeitas a influências ambientais que variam ao longo do tempo. 
Segundo, a violência psicológica (quer perpetrada, quer sofrida) parece independente da composição de género das díades, algo que os resultados anteriores já prenunciavam e que encontra paralelo no estudo de bullying entre irmãos (Duncan, 1999). De facto, as táticas psicológicas de abuso parecem ser um lugar-comum entre as estratégias de intimidação nas fratrias (ex., Wiehe, 1997). Terceiro, as díades masculinas foram as que apresentaram índices mais severos de violência física, tanto perpetrada como sofrida. Se, para alguns autores (Goodwin \& Roscoe, 1990; Roscoe et al., 1987), os atos de violência física mais graves ocorrem em díades masculinas, como aqui observado, para outros (Pepler, Abramovitch, \& Corter, 1981) vigoram sobretudo em díades masculino-feminino, particularmente se constituídas por irmãs mais novas e irmãos mais velhos (Hoffman \& Edwards, 2004). Questões metodológicas poderão estar implicadas nesta discrepância (ex., os efeitos de idade acima referidos, as dinâmicas do comportamento de denúncia), mas é razoável conjeturar uma dinâmica mais complexa em que fatores socioculturais e ambientais interajam. Por outras palavras, a exposição a fatores ambientais de risco (ex., o tempo que os irmãos passam juntos, a supervisão parental, a exposição a outras formas de violência familiar) pode variar entre culturas e gerações. Relativamente à forma sexual de violência, os dados comparativos entre díades são praticamente inexistentes, pelas razões amostrais anteriormente evocadas. Não obstante, um estudo nos EUA, cuja amostragem se baseou em mais de 10000 denúncias oficiais de ofensas sexuais entre irmãos, atribuiu mais de $60 \%$ delas a ofensores masculinos e vítimas femininas; contudo, as díades masculinas foram a segunda díade mais frequente, responsável por $25 \%$ dos registos (Krienert \& Walsh, 2011b). Importa referir que a amostragem destes dois estudos assentou em denúncias, pelo que se desconhecem dados retrospetivos de amostras comunitárias. No entanto, no presente estudo, as díades com maior frequência de comportamentos sexualmente abusivos foram coincidentes, numa magnitude inversa.

\section{Considerações Finais}

Finalmente, algumas limitações do presente estudo devem ser reconhecidas. Por um lado, um desenho transversal retrospetivo impede extrapolações de causalidade. Por outro lado, o recrutamento exclusivo de universitários impediu a generalização de resultados dada a homogeneidade amostral produzida. Todavia, nenhuma destas generalizações era pretensão deste estudo. São sobretudo instrumentais as fragilidades que aqui importa discutir. Primeiro, as instruções das CTS2-SP referem-se a episódios (eventualmente) ocorridos quando o participante tinha "aproximadamente 13 anos"; ao fazê-lo, o questionário enquadra as respostas numa espécie de "incidência retrospetiva". Este período temporal poderá ser limitativo, não só pelas complexas influências da idade na dinâmica de violência das fratrias, mas também por propiciar viés de memória (i.e., o participante evocar erroneamente episódios que julga terem ocorrido precisamente aos 13 anos, ou o inverso). Por estas razões, o período temporal de resposta deverá ser mais extenso, por exemplo, "dos 10 aos 14 anos", tal como o utilizado por Noland e colaboradores (2004). Segundo, as CTS2-SP cinge-se à conflitualidade de uma só díade fraterna (i.e., quando o sujeito tem mais do que um irmão é convidado a responder em relação ao que lhe é mais próximo em idade), o que resulta, tal como noutros estudos (ex., Kettrey \& Emery, 2006; Noland et al., 2004), na exclusão de dados sobre violência que ocorra noutras díades. Isto poderá ser particularmente preocupante no estudo da violência sexual: à luz dos dados de Krienert e Walsh (2011b), 13\% dos incidentes foram perpetrados por um ofensor a múltiplas vítimas e $10 \%$ dos registos foram perpetrados por múltiplos ofensores a uma mesma vítima; ora esta realidade escaparia às CTS2-SP. Estudos futuros deverão implementar metodologias que privilegiem o cruzamento de informação, integrando várias fontes (ex., outros familiares, professores, registos hospitalares e judiciais), uma vez que as taxas reportadas de violência entre irmãos tendem a ser superiores quando os jovens são diretamente questionados (Goodwin \& Roscoe, 1990). Por outro lado, o fenómeno carece de estudos longitudinais que abranjam diferentes faixas etárias e comparem diferentes amostras (i.e., clínicas versus comunitárias) de modo a documentar as interações ao longo do desenvolvimento das fratrias e o modo como se transpõem para as relações adultas. Necessariamente, as eventuais influências dos "novos" fatores ambientais (ex., exposição à violência na internet e redes sociais) devem também ser contempladas. Seria igualmente interessante abordar o fenómeno da violência entre irmãos junto de famílias em que um dos membros da díade fraterna é possuidor de necessidades educativas especiais, uma vez que segundo Nunes e Aiello (2008) torna-se mais complexa a análise desse relacionamento quando estamos na presença destas situações.

Em suma, este foi o primeiro estudo a estimar as taxas de ocorrência de vários tipos de violência entre irmãos numa grande amostra de estudantes universitários portugueses, caracterizando por género e composição das díades fraternas os abusos e vitimizações mais frequentes. As elevadas frequências encontradas, a par da evidente coocorrência de abusos e vitimizações, alertam para a sua reciprocidade na relação fraterna. Diversas experiências de vitimização diferiram por género, salientando a necessidade do estudo de perfis diferenciais para sustentar estratégias de prevenção, práticas profissionais e políticas institucionais.

\section{Referências}

Adler, A. (1993). El carácter neurótico (2. ed.). Barcelona, España: Paidós Ibérica. (Original publicado em 1930)

Adler, N. A., \& Schutz, J. (1995). Sibling incest offenders. Child Abuse \& Neglect, 19, 811-819.

Bank, S. P., \& Kahn, M. D. (1997). The sibling bond. New York: Basic Books. 
Relva, I. C., Fernandes, O. M., Alarcão, M. \& Martins, A. Q. (2014). Estudo Exploratório sobre a Violência entre Irmãos em Portugal.

Bograd, M. (1990). Feminist perspectives on wife abuse: An introduction. In K. Yllo \& M. Bograd (Eds.), Feminist perspectives on wife abuse (pp. 11-26). Newbury Park, CA Sage.

Button, D. M., \& Gealt, R. (2010). High risk among victims of sibling violence. Journal of Family Violence, 25(2), 131-140.

Caffaro, J. V., \& Conn-Caffaro, A. (1998). Sibling abuse trauma: Assessment and intervention strategies for children, families, and adults. New York: Haworth Press.

Caffaro, J. V., \& Conn-Caffaro, A. (2005). Treating sibling abuse families. Aggression and Violent Behaviour, 10, 604-623.

Canavan, M. M., Meyer, W. J., \& Higgs, D.C. (1992). The female experience of sibling incest. Journal of Marital and Family Therapy, 18(2), 129-142.

Cohen, J. (1992). A power primer. Psychological Bulletin, 112, 155-159.

Cyr, M., Wright, J., McDuff, P., \& Perron, A. (2002). Intrafamilial sexual abuse: Brother-sister does not differ from fatherdaughter and stepfather-step-daughter incest. Child Abuse \& Neglect, 26, 957-973.

Duncan, R. (1999). Peer and sibling aggression: An investigation of intra-and extra-familial bullying. Journal of Interpersonal Violence, 14, 871-886.

Dunn, J. (1983). Siblings relationships in early childhood. Child Development, 54, 787-811.

Eriksen, S., \& Jensen, V. (2006). All in the family? Family environment factors in sibling violence. Journal of Family Violence, 21, 497-507.

Eriksen, S., \& Jensen, V. (2009). A push or a punch: Distinguishing the severity of sibling violence. Journal of Interpersonal Violence, 24, 183-208.

Felson, R. B., \& Russo, N. F. (1988). Parental punishment and sibling aggression. Social Psychology Quarterly, 51(1), 11-18.

Felson, R. B., \& Tedeschi, J. (1993). Social interactionist perspectives on aggression and violence: An introduction. In R. Felson \& J. Tedeschi (Eds.), Aggression and violence: Social interactionist perspectives (pp. 1-10). Washington, DC: American Psychological Association.

Fernandes, O. M., Alarcão, M., \& Raposo, J. V. (2007). Posição na fratria e personalidade. Estudos de Psicologia (Campinas), 24(3), 297-304.

Ferreira, E. A. P., \& Mettel, T. P. L. (1999). Interacção entre irmãos em situação de cuidados formais. Psicologia: Reflexão e Crítica, 12(1), 133-146.

Field, A. (2009). Discovering statistics using SPSS ( $3^{\text {rd }}$ ed.). London: Sage.

Finkelhor, D. (1980). Sex among siblings: A survey of prevalence, variety, and effects. Archives of Sexual Behavior, 9, 171-194.

Finkelhor, D., Turner, H., \& Ormond, R. (2006). Kid's stuff: The nature and impact of peer and sibling violence on younger and older children. Child Abuse \& Neglect, 30(12), 1401-1421.

Garcia, M., Shaw, D., \& Yaggi, K. (2000). Destructive sibling conflict and the development of conduct problems in young boys. Developmental Psychology, 1, 44-53.

Goodwin, M. P., \& Roscoe, B. (1990). Sibling violence and agnostic interactions among middle adolescents. Adolescence, 25, 451-467.

Graham-Bermann, S., Cutler, S., Litzenberger, B., \& Schwartz, W. (1994). Perceived conflict and violence in childhood sibling relationships and later emotional adjustment. Journal of Family Psychology, 8, 85-97.
Hardy, M. S. (2001). Physical aggression and sexual behavior among siblings: A retrospective study. Journal of Family Violence, 3, 255-268.

Hardy, M. S., Beers, B., Burgess, C., \& Taylor, A. (2010). Personal experience and perceived acceptability of sibling aggression. Journal of Family Violence, 25, 67-71.

Hoffman, K. I., \& Edwards, J. N. (2004). An integrated theoretical model of sibling violence and abuse. Journal of Family Violence, 3, 185-201.

Hoffman, K. I., Kiecolt, K. J., \& Edwards, J. N. (2005). Physical violence between siblings: A theoretical and empirical analysis. Journal of Family Issues, 26, 1103-1130.

Kettrey, H., \& Emery, E. (2006). The discourse of sibling abuse. Journal of Family Violence, 21, 407-416.

Kiselica, M., \& Morrill-Richards, M. (2007). Sibling maltreatment: The forgotten abuse. Journal of Counseling \& Development, 85, 148-160.

Krienert, J. L., \& Walsh, J. A. (2011a). My brother's keeper: A contemporary examination of reported sibling violence using national level data, 2000-2005. Journal of Family Violence, 26, 331-342.

Krienert, J. L., \& Walsh, J. A. (2011b). Sibling sexual abuse: An empirical analysis of offender, victim, and event characteristics in National Incident-Based Reporting System (NIBRS) data, 2000-2007. Journal of Child Sexual Abuse, 20, 353-372.

Laurance, J. (2000, November 19). Revealed: The truth about child sex abuse in Britain's families. Child maltreatment in the United Kingdom. The Independent on Sunday.

Leder, J. (1993). Adult sibling rivalry. Psychology Today, 26, 56-62.

Lytton, H., \& Romney, D. M. (1991). Parents' differential socialization of boys and girls: A meta-analysis. Psychological Bulletin, 109, 267-296.

Minuchin, S. (1982). Famílias, funcionamento \& tratamento. Porto Alegre, RS: Artes Médicas. (Original publicado em 1974)

Noland, V. J., Liller, K. D., McDermott, R. J., Coutler, M. L., \& Seraphine, A. E. (2004). Is adolescent sibling violence a precursor to college dating violence? American Journal of Health Behavior, 28, 13-23.

Noller, P. (2005). Sibling relationships in adolescence: Learning and growing together. Personal Relationships, 12(1), 1-22.

Nunes, C. L., \& Aiello, A. L. (2008). Interação entre irmãos: Deficiência mental, idade e apoio social da família. Psicologia: Reflexão e Crítica, 21(1), 42-50.

O’Brien, M. (1991). Taking sibling incest seriously. In M. Patton (Ed.), Understanding family sexual abuse. Newbury Park, CA: Sage.

Peat, J., \& Barton, B. (2005). Medical statistics: A guide to data analysis and critical appraisal. Harayana, India: BMJ Books.

Pepler, D., Abramovitch, R., \& Corter, C. (1981). Sibling interaction in the home: A longitudinal study. Child Development, 52, 1344-1347.

Relva, I. C., Fernandes, O., \& Costa, R. (2013). Psychometric properties of Revised Conflict Tactics Scales: Portuguese sibling version (CTS2-SP). Journal of Family Violence, 28(6), 577-585. doi:10.1007/s10896-013-9530-0

Roscoe, B., Goodwin, M. P., \& Kennedy, D. (1987). Sibling violence and agonistic interactions experienced by early adolescents. Journal of Family Violence, 2, 121-137.

Rothman, E., Johnson, R., Azrael, D., Hall, D. H., \& Weinberg, J. (2010). Perpetration of physical assault against dating partners, peers, and siblings among a locally representative sample 
of high school students in Boston, Massachusetts. Archives of Pediatrics \& Adolescent Medicine, 164(12), 1118-1124.

Rudd, J. M., \& Herzberger, S. D. (1999). Brother-sister incest father-daughter incest: A comparison of characteristics and consequences. Child Abuse \& Neglect, 23, 915-928.

Russell, D. (1986). The secret trauma: Incest in the lives of girls and women. New York: Basic Books.

Seto, M. C., \& Lalumière, M. L. (2010). What is so special about male adolescent sexual offending? A review and test of explanations through meta-analysis. Psychological Bulletin, 136(4), 526-575.

Simonelli, C. J., Mullis, T., Elliot, A. N., \& Pierce, T. W. (2002). Abuse by siblings and subsequent experiences of violence within the dating relationship. Journal of Interpersonal Violence, 17, 103-121.

Smith, H., \& Israel, E. (1987). Sibling incest: A study of the dynamics of 25 cases. Child Abuse \& Neglect, 11, 101-108.

Snyder, J., Bank, L., \& Burraston, B. (2005). The consequences of antisocial behavior in older male siblings for younger brothers and sisters. Journal of Family Psychology, 19, 643-653.

Steinmetz, S. K. (1977). The cycle of violence, assertive, aggressive, and abusive family interaction. New York: Praeger.

Straus, M. A., Gelles, R. J., \& Steinmetz, S. K. (1980). Behind closed doors. Violence in the american family. Garden City, NY: Anchor Books.

Straus, M. A., Hamby, S. L., Boney-McCoy, S., \& Sugarman, D. B. (1996). The revised Conflict Tactics Scales (CTS2): Development and preliminary psychometric data. Journal of Family Issues, 17, 283-316.

Tabachnick, B. G., \& Fidell, L. S. (2007). Using multivariate statistics ( $5^{\text {th }}$ ed.). Boston, MA: Allyn and Bacon.

Toman, W. (1959). Family constellation as a basic personality determinant. Journal of Individual Psychology, 18, 48-51.

Toman, W. (1993). Family constellation: Its effects on personality and social behavior. New York: Springer.

Welfare, A. (2008). How qualitative research can inform clinical interventions in families recovering from sibling sexual abuse. ANZ Journal of Family Therapy 29(5), 139-147.

Wiehe, V. R. (1997). Sibling abuse: Hidden physical, emotional and sexual trauma ( $2^{\text {nd }}$ ed.). Thousand Oaks, CA: Sage. 